\title{
Estimación del coeficiente de capturabilidad (q) y de la biomasa de anguila común (Ophichthus remiger) del norte de Perú mediante análisis geoestadístico de las densidades de captura
}

\author{
Catchability and Biomass estimation of common eel (Ophichthus remiger) off northern Peru by \\ using likelihood-based geostatistical method on fish density
}

\author{
Jimmy Juan Martina Vásquez ${ }^{1}$
}

\begin{abstract}
Resumen
El objetivo del presente estudio fue estimar la biomasa mensual de anguila común $(O$. remiger) del norte de Perú utilizando geoestadística de verosimilitud aplicada a valores de densidad de captura a partir de 13092 lances de pesca de embarcaciones artesanales (15 m de eslora) que operaron regularmente desde enero 2003 hasta diciembre 2007 , latitudinalmente desde $4^{\circ} \mathrm{S}$ hasta $7^{\circ} \mathrm{S}$ y batimétricamente desde 80 hasta $250 \mathrm{~m}$ de profundidad. La biomasa, cpue y el coeficiente de capturabilidad son calculados cada uno en forma independiente. Se evalúa cada área de lance de pesca con trampas como si cada una fuera un arrastre. Conociendo la densidad en la zona de pesca en función al total de capturas y áreas de lance de pesca $\left(\mathrm{t} / \mathrm{km}^{2}\right)$, se modela su distribución espacial con geoestadística de verosimilitud. Mediante kriging, se estima la densidad del recurso en el área de prospección. La media del kriging equivale a la densidad media del recurso; luego, el producto de ésta con el área efectiva del stock en el caladero es la biomasa relativa. Se consideró como área efectiva del stock solo las zonas donde se obtuvieron capturas dentro del área de prospección, permitiendo una estimación más realista del hábitat del recurso. La cpue promedio de $O$. remiger para todo el periodo de estudio fue 0.34 t/lance (coeficiente de variabilidad (c.v.) = 20.6\%), el mínimo valor fue $0.21 \mathrm{t}$ /lance en enero de 2007 y el máximo de 0,56 t/lance en diciembre de 2007, año en el que ocurren dos cambios de caladeros. La biomasa promedio de $O$. remiger en todo el periodo fue $921 \mathrm{t}$ (c.v. $=52 \%$ ). El mínimo valor fue $273 \mathrm{t}$ en abril de 2004 y el máximo de 2644 t en mayo de 2007.
\end{abstract}

Palabras clave: Biomasa; captura por unidad de esfuerzo (cpue); coeficiente de capturabilidad; anguila común (Ophichthus remiger); geoestadística; verosimilitud.

\begin{abstract}
The main objective of this study was to estimate monthly biomass of common eel (O. remiger) off northern Peru by means of using likelihood-based geostatistical method on fish density from 13092 fishing task, they were performed by artisanal fishing vessels ( $15 \mathrm{~m}$ length) from January 2003 to December 2007 , from latitude $4^{\circ}$ to $7^{\circ} \mathrm{S}$, and bathymetrically from 80 to $250 \mathrm{~m}$ depth. Biomass, cpue and catchability coefficient were each computed independently. Each hunting fishing area was calculated as if it was a hauling fishing area by making an analogy described in this publication. Spatial distribution of $O$. remiger is modeled by likelihood geostatistics from the fishing density $\left(\mathrm{t} / \mathrm{km}^{2}\right)$. Simple $\mathrm{kriging}$ is used to calculate mean density; by the product of this with effective stock area the relative biomass is finally computed. Effective stock area is defined as the one where catches of $O$. remiger in the survey area were registered. It allows having a better estimation of the area where $\mathrm{O}$. remiger can be found. This provided an estimate of $\mathrm{O}$. remiger average biomass at $921 \mathrm{t}(\mathrm{c} . \mathrm{v} .=72 \%)$, the minimum value was at $273 \mathrm{t}$ in April 2004 and the maximum was at $2644 \mathrm{t}$ in May 2007. The average of catch per unit of effort (cpue) was estimated at $0.34 \mathrm{t} /$ haul (coefficient of variation (c.v.) $=20.6 \%$ ), the minimum value was at $0.2 \mathrm{t} /$ haul in January 2007 and the maximum at $0.56 \mathrm{t} /$ haul in December 2007 when the fleet visited other fishing ground. Keywords: Biomass, catch per unit of effort (cpue), catchability coefficient, common eel (Ophichthus remiger), geostatistical method, likelihood.
\end{abstract}

Keywords: Biomass; catch per unit of effort (cpue); catchability coefficient; common eel (Ophichthus remiger); geostatistical method; likelihood.

\section{Introducción}

$O$. remiger es uno de los principales recursos demersales capturado en el norte de Perú, cuyo destino, por más de veinte años, es exclusivamente para exportación a mercados asiáticos (PROMPEX, 2016). Referente a esta pesquería, destacan Castillo et al. (2000) y Castillo \& Gómez (2002), quienes mencionan que en invierno (julio a setiembre) y primavera (octubre a diciembre) como las épocas con mayores capturas, coincidiendo con lo informado por el Ministerio de Producción (PRODUCE, 2008) y el Instituto del Mar del Perú (IMARPE, 2006). Posteriormente, PRODUCE (2010) reglamenta la pesquería de $O$. remiger en Perú, estableciendo la talla mínima de captura en 42 $\mathrm{cm}$ longitud total con registros de desembarques en Paita 
$\left(05^{\circ} 04^{\prime} \mathrm{S}, 81^{\circ} 06^{\prime} \mathrm{W}\right)$ y Sechura $\left(05^{\circ} 45^{\prime} \mathrm{S} ; 80^{\circ} 48^{\prime} \mathrm{W}\right)$.

Se ha señalado que entre la cpue y la biomasa existe una relación relativamente simple (Arreguín-Sánchez, 1996; Quinn \& Deriso, 1999) de la forma:

$$
\text { cpue }_{t}=q_{t} B_{t}
$$

donde: cpue es la captura por unidad de esfuerzo ( $\mathrm{t} /$ lance), $q$ es el coeficiente de capturabilidad (lance $\left.{ }^{-1}\right), B$ es la biomasa ( $\mathrm{t}$ ), $t$ es el período de tiempo (mes, en este trabajo).

Se define como lance de pesca a la colocación de la línea de pesca en el agua, contiene entre 800 a 1200 trampas separadas cada 5 metros aproximadamente y con un tiempo de reposo de tres horas.

El coeficiente de capturabilidad $(q)$ corresponde a la eficiencia del arte de pesca (Neis et al., 1999) y se define como la capacidad para capturar peces o la fracción de peces capturados por unidad de esfuerzo (ArreguínSánchez, 1996; Cadima, 2003; Marchal et al., 2003; Wilberg et al., 2010). La eficiencia del arte de pesca está en función de cambios en la tecnología de pesca (poder de pesca), comportamiento del pescador (Chávez, 2000), factores ambientales y agregación reproductiva del recurso objetivo (Arreguín-Sánchez, 1996). Debido a la complejidad que implica estimar $q$, muchas veces se asume que es constante o igual a 1 . Sin embargo, varios trabajos han mostrado que $q$ no es constante en peces pelágicos (Ahrens \& Walters, 2005), en peces demersales (Yousif \& Sabrah, 2005; Bahamon et al., 2009), en crustáceos (Dichmont et al., 2003) o moluscos (Chávez, 2000).

En este trabajo se estima la densidad $\left(\mathrm{t} / \mathrm{km}^{2}\right)$ y el área efectiva de distribución del stock $\left(\mathrm{km}^{2}\right)$ a partir de capturas geo-referenciadas, ya que del producto entre ambas puede obtenerse la biomasa (Roa-Ureta \& Niklitschek, 2007). Se aplica geoestadística de verosimilitud para determinar el modelo de estructura espacial de los lances y capturas.

El análisis espacial de capturas con geoestadística ayuda a interpretar la conducta del pescador, que no es aleatoria en el sentido que el pescador permanece en un caladero en función del éxito de sus capturas (Walters, 2003; Walters \& Hilborn, 2005).

En algunos estudios de evaluación de recursos pesqueros con análisis espacial de las capturas, se ignora que puedan existir subáreas cercanas donde no se realiza lances (Walters, 2003), sea por zonas inaccesibles (rocosas) o con accidentes geográficos. En consecuencia, en el presente trabajo se usa el concepto de área efectiva del stock, que se definirá más adelante.

Arreguín-Sánchez (1996) y Quinn \& Deriso (1999) mencionan la biomasa y la cpue se relacionan de la forma:

$$
\text { cpue }_{t}=q_{t} B_{t}
$$

donde: cpue es la captura por unidad de esfuerzo (t/ lance), $q$ es el coeficiente de capturabilidad (lance $\left.{ }^{-1}\right), B$ es la biomasa ( $\mathrm{t}$ ), $t$ es el período de tiempo (mes, en este trabajo).

En forma independiente de la cpue, las estimaciones de biomasa en diversas pesquerías son necesarias para identificar tendencias ante diferentes niveles de explotación que permitan la pesca sostenible, esto debido a los casos de hiperestabilidad o hiperdepleción (Gaertner \& DreyfusLeón, 2004; Harley et al., 2001).

Durante el año 2006 el Instituto del Mar de Perú consideró cuantificar la biomasa de $O$. remiger, desarrollando investigaciones continuas. Para el tercer trimestre del año 2016 se ha reportado que la captura por unidad de esfuerzo (cpue) presentó variaciones de 0,162 a $1,521 \mathrm{~kg} / \mathrm{trampa}$ (tr) y un promedio de $0,635 \mathrm{~kg} /$ tr (PRODUCE, 2016). Por lo tanto, el presente trabajo pretende contribuir al conocimiento del stock y el tipo de distribución espacial del recurso, presentando valores de biomasa con ayuda de geoestadística. Con este procedimiento los evaluadores tendrán una herramienta adicional para diagnosticar al stock en forma más precisa y contribuir a una mejor conservación del recurso.

\section{Materiales y métodos}

Se realizaron 13092 lances de cruceros entre $03^{\circ} 21^{\prime} \mathrm{S}$ y $07^{\circ} 00^{\prime} \mathrm{S}$, frente a la costa norte de Perú, desde enero 2003 hasta diciembre 2007. Se trabajó con información de estas capturas diarias geo-referenciadas obtenidas a partir de información de bitácoras de cuatro a ocho naves artesanales, cuyas dimensiones promedio son: $11 \mathrm{~m}$ de eslora; 2,5 m de manga; 1,5 m de puntal; capacidad de bodega entre 4 y $6 \mathrm{t}$.

Para procesar preliminarmente los datos de capturas geo-referenciadas se utilizaron los programas Surfer 8.0 y Excel. Luego se utilizó el sistema de programación estadística R, usando los paquetes PBSmapping, geoRglm, car y nortest para reconstruir la distribución de las capturas por caladero.

En un lance de pesca, la densidad $D_{k, t}$ en el caladero o zona de pesca $k$ en un tiempo de reposo del arte $t$ es el cociente:

$$
D_{k, t}=\frac{C_{k, t}}{\mathrm{a}_{k, t}}
$$

donde $C_{k, t}$ es la captura. El área efectiva $\mathrm{a}_{k, t}$ en un lance de pesca en el caladero $k$ al tiempo $t$ se calcula como la suma de la mitad de una circunferencia de radio $r_{e f . L}$, un rectángulo de lado $L$ y otra mitad de una circunferencia también de radio $r_{\text {ef.L }}$, según la siguiente expresión:

$$
a_{k, t}=L 2 r_{e f . L}+\pi \cdot r_{e f . L}^{2}
$$

donde $L$ es la longitud de la línea de pesca; $r_{\text {ef. } L}$ es el radio efectivo del lance, que se asume $30 \mathrm{~m}$ para cada trampa (Matsumiya \& Imai, 1987), según se indica en la Figura 1. Aat es el área de acción de cada trampa en el 


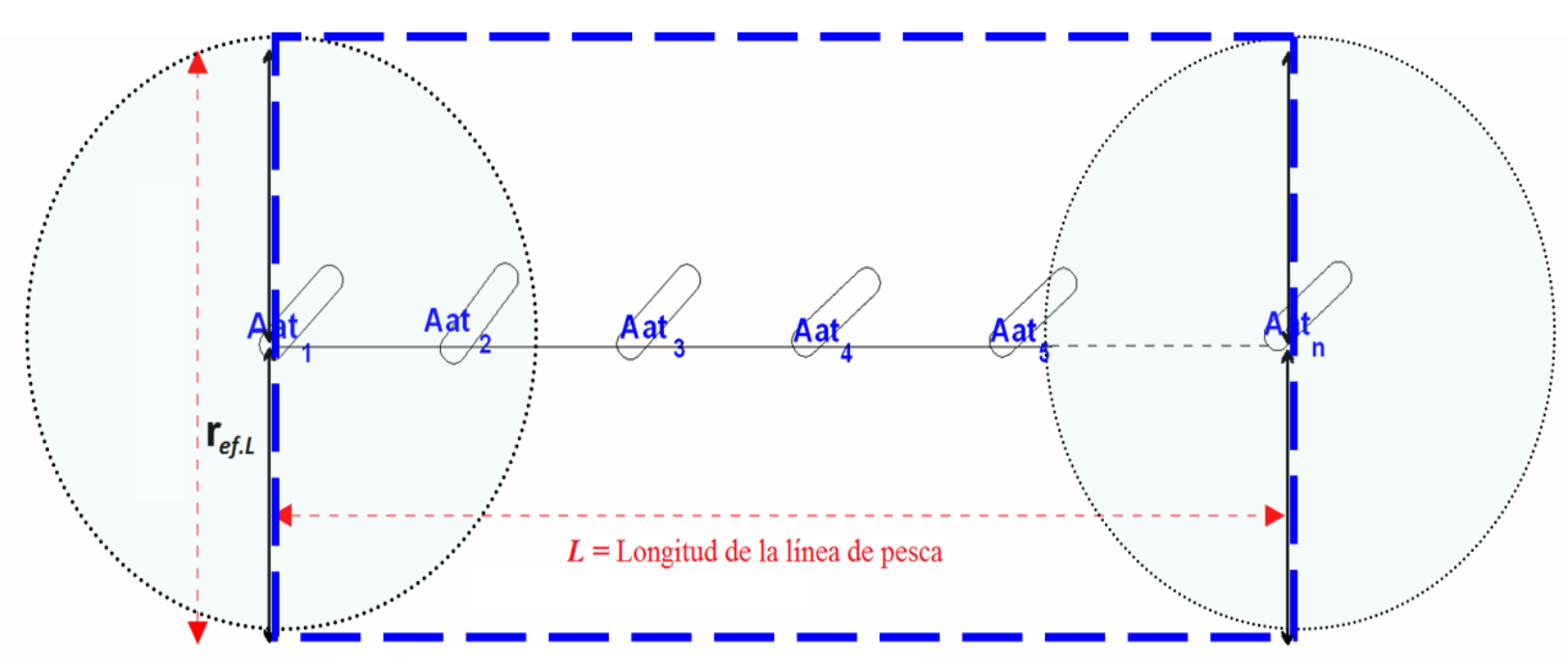

Figura 1. Representación del área efectiva del lance de pesca $\quad \mathrm{a}_{k, t}$ en la zona de pesca o caladero $k$ a un tiempo de reposo del arte $t$, como la sumatoria del área de acción Aat de cada trampa en el agua

agua, partiendo del supuesto que la corriente tiene un efecto despreciable.

En el presente estudio, se considera que la eficiencia de cada trampa es $100 \%$, esto es, que todos los ejemplares de anguila común que se encuentran en el área de la trampa Aat son atraídos y capturados (no hay escape). También se asume que ningún ejemplar disponible mayor de $42 \mathrm{~cm}$ logra escapar del aparejo de pesca. A partir de los datos georeferenciados de densidad por lance $\left(\mathrm{t} / \mathrm{km}^{2}\right)$, con ayuda del criterio Akaike, se identificaron los variogramas que mejor describen la distribución espacial del recurso, ya sean de tipo exponencial, gausiano, esférico u otro según RoaUreta \& Niklitschek (2007). Adicionalmente, los mismos autores mencionan que la probabilidad de observar el recurso en cada lance se relaciona con un proceso gausiano no observado mediante la función de enlace logit, según la siguiente expresión:

$$
g(p)=\ln [p(1-p]
$$

donde $p$ la probabilidad de observar el stock en el modelo espacial y la constante $g(p)$ es la media del proceso de observación binomial en un modelo lineal generalizado espacial. Diggle et al. (2003) proponen utilizar la Cadena de Markov Monte Carlo (MCMC) para simular la distribución condicional de un variograma ordinario de data tipo binomial-espacial dadas las observaciones positivas en una cuadrícula y usar las simulaciones aproximándolas a la función de verosimilitud. La cadena de Markov se obtiene de una distribución condicional binomial-espacial, cuya media a estimar es $g(p)$.

Roa-Ureta \& Niklitschek (2007) señalan que la probabilidad de observar el stock en el modelo espacial $(p)$ se obtiene de re-transformar la función de enlace binomial, esto es:

$$
\frac{e^{g(p)}}{1+e^{g(p)}}=p
$$

donde $\mathrm{g}(p)$ es la función de enlace binomial.

Para el análisis geoestadístico de verosimilitud se usó el paquete geoRglm desarrollado por Christensen \& Ribeiro (2002) en el programa R. Para esto, Diggle et al. (2003), Roa-Ureta \& Niklitschek (2007) y Horton \& Kleinman (2011) recomiendan normalizar las densidades de cada lance $\left(D_{k, t}\right)$ mediante la transformación con la función $\mathrm{f}$, donde el parámetro $\lambda$ normaliza las observaciones (Regalado \& Ritter, 2005). La función $\mathrm{f}_{\lambda}$ pertenece a la familia Box-Cox. Para detalles, ver Roa-Ureta \& Niklitschek (2007). En caso que se observe anisotropía, Alarcón (2003) sugiere considerar el variograma en la dirección de mayor continuidad espacial.

El área efectiva del stock ( $A_{k, t}$ ) es el área ocupada por el stock dentro del perímetro de evaluación sin incluir la batimetría rocosa, permitiendo la estimación más realista del hábitat (Roa-Ureta \& Niklitschek, 2007), según:

$$
A_{k, t}=a p
$$

donde: $A_{k, t}$ es el área efectiva del stock en el caladero $k$ y mes $t\left(\mathrm{~km}^{2}\right)$; $a$ es el área de prospección donde se realizan las capturas en $\mathrm{km}^{2}$ (donde $a=\sum_{t=1}^{n} \mathrm{a}_{k, t}, n=$ número de lances en el mes $t$ y caladero $k$ ) y $\hat{p} \quad$ es el estimador de máxima verosimilitud para la probabilidad de captura.

Finalmente, para calcular la biomasa (relativa) en $A_{k, t}$ se siguió a Aitchison (1955); fide Roa-Ureta \& Niklitschek, (2007), quien presenta una generalización para el cálculo de la media y la varianza de la biomasa, según la siguiente expresión: 


$$
B_{A_{k, t}}=A_{k, t} \theta
$$

donde: $B_{A_{k, t}}$ es la biomasa (relativa) en el área efectiva del stock; $A_{k, t}$ es el área efectiva del stock; $\theta$ es la densidad media o media del kriging. La varianza de la biomasa es estimada por:

$$
V\left(B_{A_{k, t}}\right)=a^{2}\left[\hat{p}^{2} v(\theta)+\theta^{2} v(\hat{p}]\right.
$$

donde: $a$ y $\hat{p}$ ya fueron definidos; $v(\theta)$ es la varianza del kriging; $v(\hat{p})$ es la varianza de la probabilidad de captura. La cpue promedio del mes $t$ (peso/lance) se obtiene según:

$$
\text { cpue }_{k, t}=\frac{C_{k, t}}{\Sigma L_{k, t}}
$$

donde: $C_{k, t}$ es la captura en el caladero $k$ y mes $t$; $\Sigma L_{k, t}$ es el número de lances en el caladero $k$ durante el mes $t$. El coeficiente de capturabilidad $(q)$ se estima mediante la siguiente expresión (Pérez \& Defeo, 2003):

$$
q_{k, t}=\frac{\mathrm{a}_{k, t}}{A_{k, t}}
$$

donde $\mathrm{a}_{k, t}$ es el área efectiva del lance de pesca en el caladero $k$ durante el mes $t\left(\mathrm{~km}^{2} /\right.$ lance $) ; A_{k, t}$ es el área efectiva del stock $\left(\mathrm{km}^{2}\right)$.

\section{Resultados y discusión}

\section{Resultados}

Los lances de pesca para la prospección del recurso $O$. remiger se realizaron principalmente frente al departamento de Piura, al norte de Perú (Figura 2).

La distribución de los lances de pesca muestra que las naves operaron entre las latitudes $4^{\circ}$ y $5^{\circ} \mathrm{S}$ desde enero 2003 hasta enero 2007. De febrero a diciembre de 2007 la flota amplió su distribución hacia el sur hasta $7^{\circ} \mathrm{S}$ (Figura $3)$.

Las capturas y el esfuerzo de pesca son mostrados en la Figura 4 y Tabla 1. Desde el año 2003 hasta 2007 tanto las capturas (promedio $=74,8$ t; c.v. $=41,7 \%$ ) como el esfuerzo (promedio $=217$ lances; d.s. $=77,1$ lances; c.v. $=35,4 \%$ ) presentaron alta variabilidad entre meses, manteniendo tendencias similares hasta mediados de 2006. Se distinguen tres períodos, el primero durante todo el año 2003, cuando el esfuerzo disminuye de 190 a 85 lances; las capturas siguen la misma tendencia, cayendo de 89 a menos de 40 t. Probablemente este tiempo fue de aprendizaje de las tripulaciones en la pesquería de $O$. remiger en el norte de Perú.

El segundo período es de incremento casi continuo de las capturas y del esfuerzo de pesca hasta junio de 2006, cuando el esfuerzo alcanzó hasta 224 lances. Las capturas respondieron favorablemente al esfuerzo, llegando hasta $108 \mathrm{t} / \mathrm{mes}$.

El tercer período abarca desde junio 2006 hasta diciembre 2007, cuando $O$. remiger parece no responder al incremento del esfuerzo de pesca. Desde julio hasta octubre de 2006 el esfuerzo se incrementa de 220 a 420 lances,

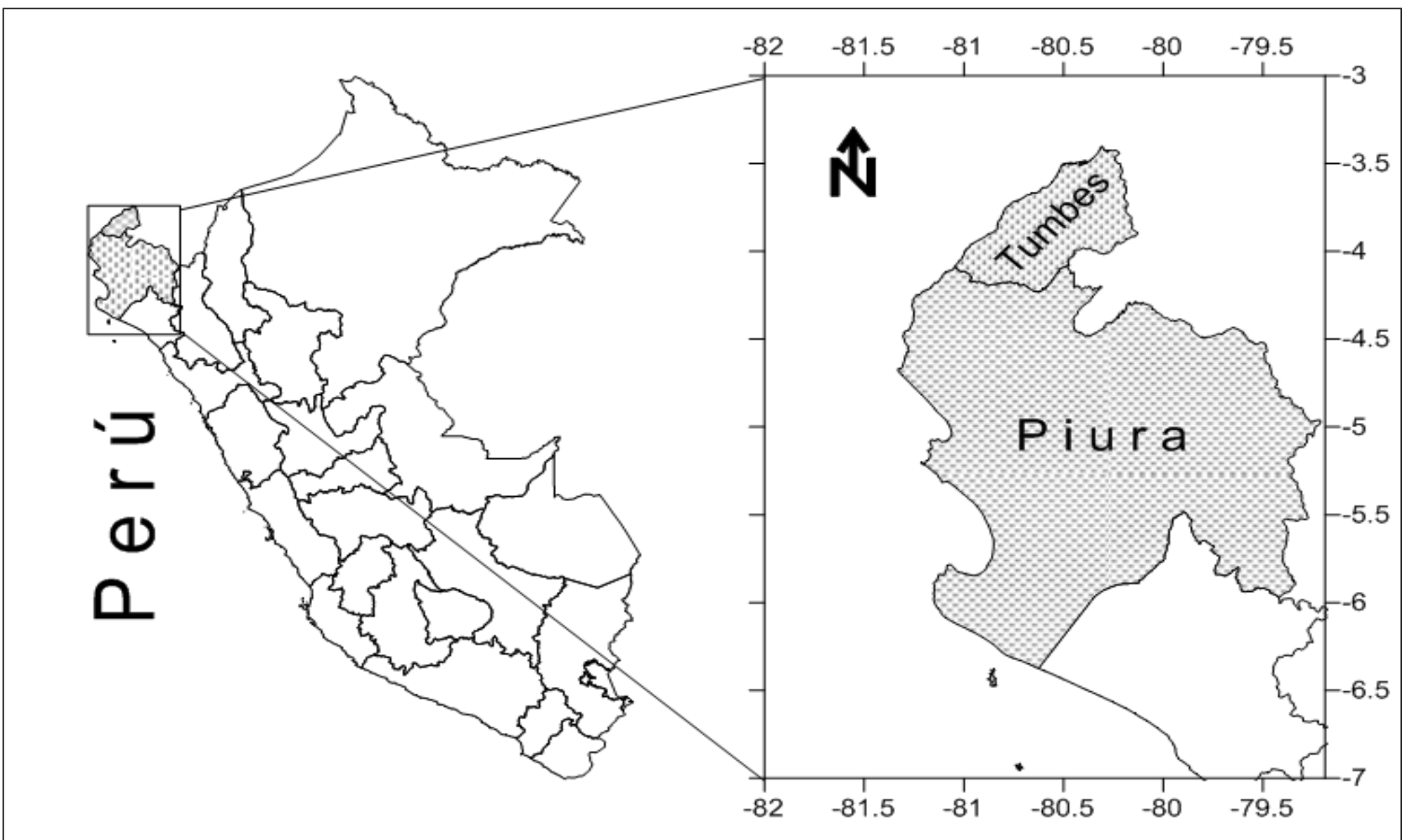

Figura 2. Localización de la zona de estudio, frente a la costa de los departamentos de Piura y Tumbes, en el norte de Perú 
respectivamente. No obstante, las capturas no superan las $90 \mathrm{t} / \mathrm{mes}$. En la primera mitad de 2007 el esfuerzo se mantuvo cerca de 320 lances (c.v. $=10 \%$ ), aunque las capturas alcanzaron a $157 \mathrm{t}$, que es el mayor valor del periodo 2003 a 2007, lo que se debería a la expansión del caladero (Figura 3).

La cpue (promedio $=0,344 \mathrm{t} /$ lance; c.v. $=22 \%$ ) y el coeficiente de capturabilidad (promedio $=6 \times 10^{-4}$ lance $^{-1}$; c.v. $=51 \%$ ) presentaron alta variabilidad en todo el periodo de estudio (Figura 5 y Tabla 2). La cpue exhibe una tendencia general decreciente por dos años, desde fines 2003 hasta agosto 2005. Seguidamente, la cpue se incrementa fuertemente hasta mitad de 2006, aunque con fluctuaciones. Sin embargo, $q$ exhibe una relación opuesta con fuertes oscilaciones con tendencia general creciente hasta mitad de 2004, cayendo luego fuerte y rápidamente (hasta octubre 2004), seguido por una recuperación leve (hasta mitad de 2005); luego mantiene una caída sostenida casi sin variaciones hasta fines de 2007 , cuando se termina la serie de datos.

La relación entre cpue y $q$ no es significativa en la pesquería de $O$. remiger del norte de Perú cuando se analiza sobre datos mensuales en el período 2003 a 2007 (Figura 6). En general, cuando el análisis de la relación entre cpue y $q$ se realiza para cada año no se revela una sola tendencia. No obstante, en los años 2004 y 2007 se obtuvo relaciones significativas entre ellas $(P<0,05)$.

En la Figura 7 se muestran datos crudos en (a) y (b), en el primero se observan la distribución espacial de los lances de pesca del recurso en estudio, mientras que en (b) se aprecia el variograma nube sin ninguna edición de sensibilización o delimitación de distancias. Se observa que las densidades presentan el efecto nugget, es decir la línea que representa esta distribución se mantiene horizontal, aproximadamente a $10 \mathrm{~m}$.

En (c) se observan los variogramas direccionales cada $45^{\circ}$, en el que se aprecia mejor la interdependencia de las densidades mientras están a distancias menores de $5 \mathrm{~km}$. En (d), el área efectiva del stock $A_{k, t} \mathrm{y}$ distribución de densidades del recurso luego del modelamiento espacial. Este procedimiento fue seguido para cada mes del período evaluado. Por motivos de espacio se muestra aquí solo para setiembre del 2007, mes en el que los variogramas direccionales fueron mejor observables.
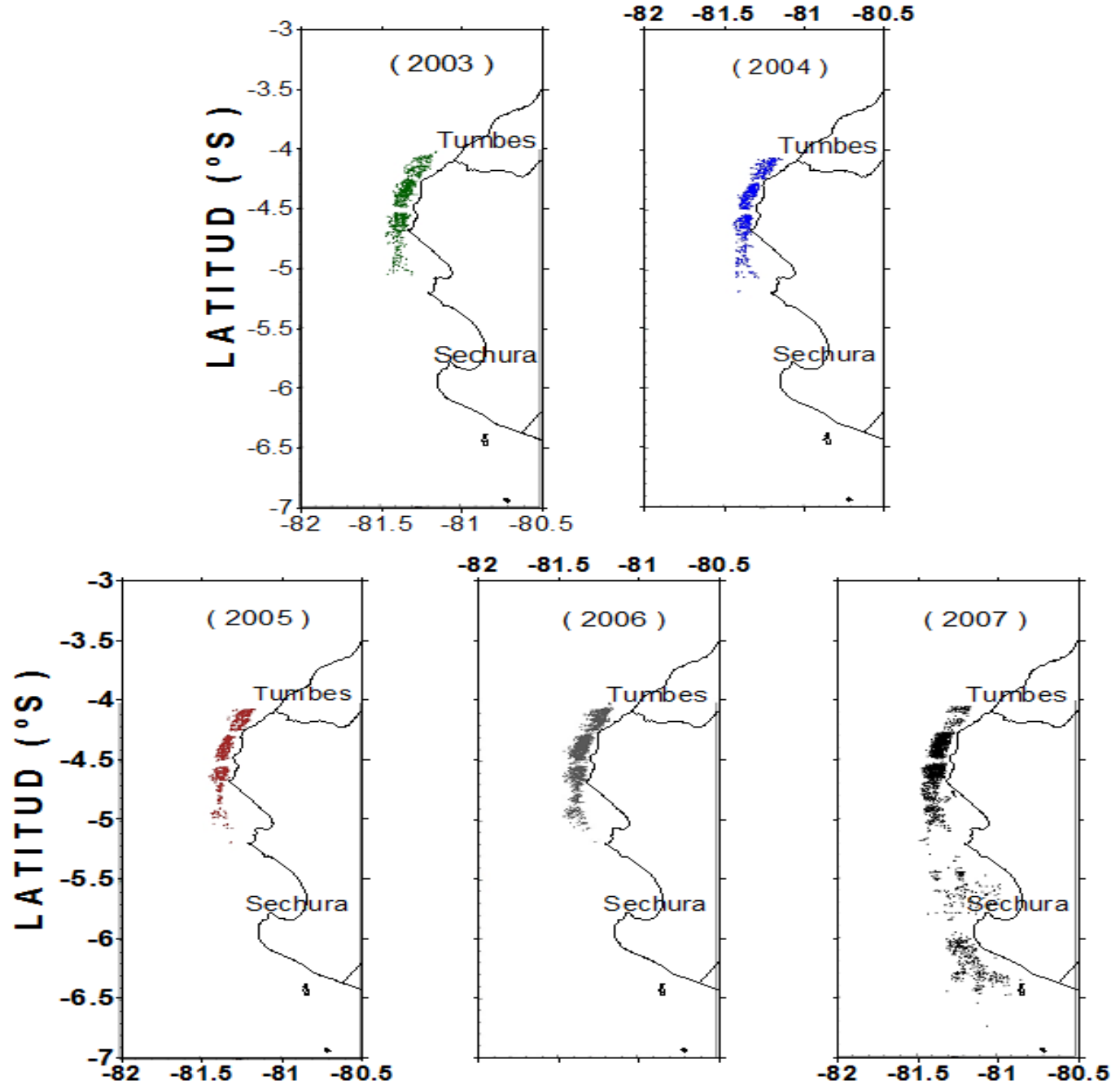

L O N G I T U D $\left({ }^{\circ} \mathbf{W}\right)$

Figura 3. Lances de pesca anuales de $O$. remiger en el norte de Perú. Período 2003 a 2007 
Tabla 1. Registros máximos y mínimos de capturas mensuales y número de lances promedio por mes. Período 2003 a 2007

\begin{tabular}{lcccccccccc}
\hline & \multicolumn{2}{c}{2003} & \multicolumn{2}{c}{2004} & \multicolumn{2}{c}{2005} & \multicolumn{2}{c}{2006} & \multicolumn{2}{c}{2007} \\
\hline & $\begin{array}{c}\text { captura } \\
(\mathrm{t})\end{array}$ & $\begin{array}{c}\text { esfuerzo } \\
\text { (lances) }\end{array}$ & $\begin{array}{c}\text { captura } \\
(\mathrm{t})\end{array}$ & $\begin{array}{c}\text { esfuerzo } \\
\text { (lances) }\end{array}$ & $\begin{array}{c}\text { captura } \\
(\mathrm{t})\end{array}$ & $\begin{array}{c}\text { esfuerzo } \\
(\text { lances })\end{array}$ & $\begin{array}{c}\text { captura } \\
(\mathrm{t})\end{array}$ & $\begin{array}{c}\text { esfuerzo } \\
(\text { lances) }\end{array}$ & $\begin{array}{c}\text { captura } \\
(\mathrm{t})\end{array}$ & $\begin{array}{c}\text { esfuerzo } \\
(\text { lances) }\end{array}$ \\
\hline Máximo & 88,8 & 200 & 71,6 & 210 & 68,3 & 217 & 108,1 & 419 & 157,3 & 347 \\
Mínimo & 41,8 & 126 & 27,1 & 85 & 41,5 & 158 & 66,9 & 220 & 69,3 & 251 \\
Promedio & 68,5 & 166,8 & 44,6 & 140,5 & 53,1 & 189 & 90,1 & 283,9 & 117,5 & 308,1 \\
c.v. (\%) & 22,2 & 16,0 & 30,8 & 31,9 & 16,7 & 9,1 & 14,5 & 22,4 & 23,3 & 9,5 \\
\hline
\end{tabular}

Tabla 2. Valores máximos y mínimos de la captura por unidad de esfuerzo (cpue en t/lance) y coeficiente de capturabilidad $\left(\mathrm{q}\right.$ en lance $\left.{ }^{-1}\right)$, y promedio por mes. Período 2003 a 2007

\begin{tabular}{lcccccccccc}
\hline & \multicolumn{2}{c}{2003} & \multicolumn{2}{c}{2004} & \multicolumn{2}{c}{2005} & \multicolumn{2}{c}{2006} & \multicolumn{2}{c}{2007} \\
\hline & cpue & $\mathrm{q}^{*} 10^{-4}$ & cpue & $\mathrm{q}^{*} 10^{-4}$ & cpue & $\mathrm{q}^{*} 10^{-4}$ & cpue & $\mathrm{q}^{*} 10^{-4}$ & cpue & $\mathrm{q}^{*} 10^{-4}$ \\
\hline Máximo & 0,467 & 8,5 & 0,397 & 14,1 & 0,323 & 8,1 & 0,482 & 5,9 & 0,566 & 3,0 \\
Mínimo & 0,331 & 5,1 & 0,235 & 4,4 & 0,243 & 5,6 & 0,216 & 1,8 & 0,211 & 1,8 \\
Promedio & 0,406 & 6,7 & 0,322 & 8,5 & 0,280 & 6,8 & 0,331 & 3,8 & 0,382 & 2,1 \\
c.v. (\%) & 10,0 & 14,9 & 14,2 & 38,8 & 9,2 & 10,2 & 25,6 & 36,8 & 23,5 & 14,2 \\
\hline
\end{tabular}

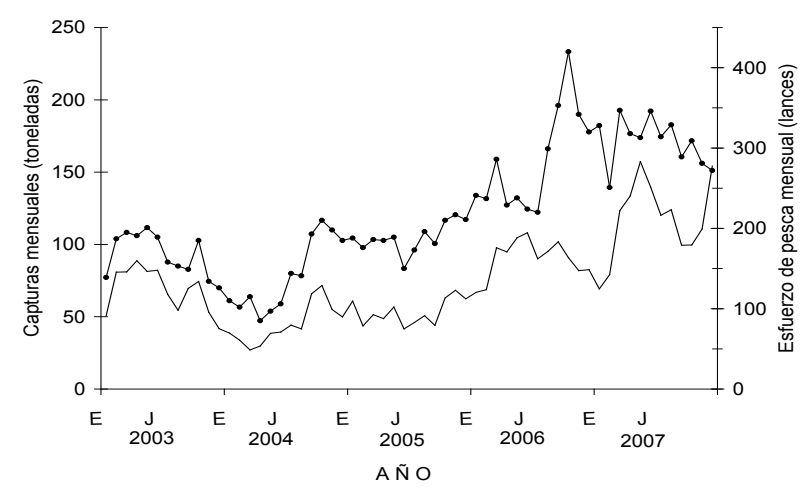

Figura 4. Capturas mensuales (línea continua) y esfuerzo mensual (línea con círculos) en la pesquería de $O$. remiger del norte de Perú

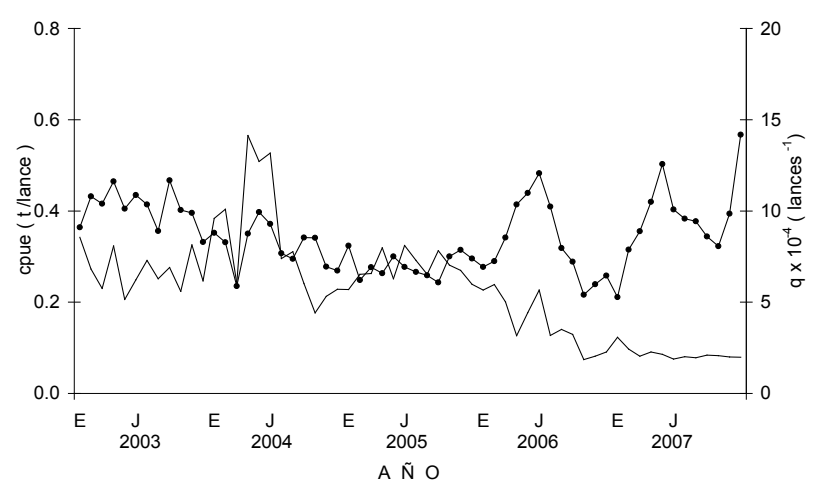

Figura 5. Captura por unidad de esfuerzo o cpue (puntos) y coeficiente de capturabilidad (línea) en la pesquería de $O$. remiger del norte de Perú

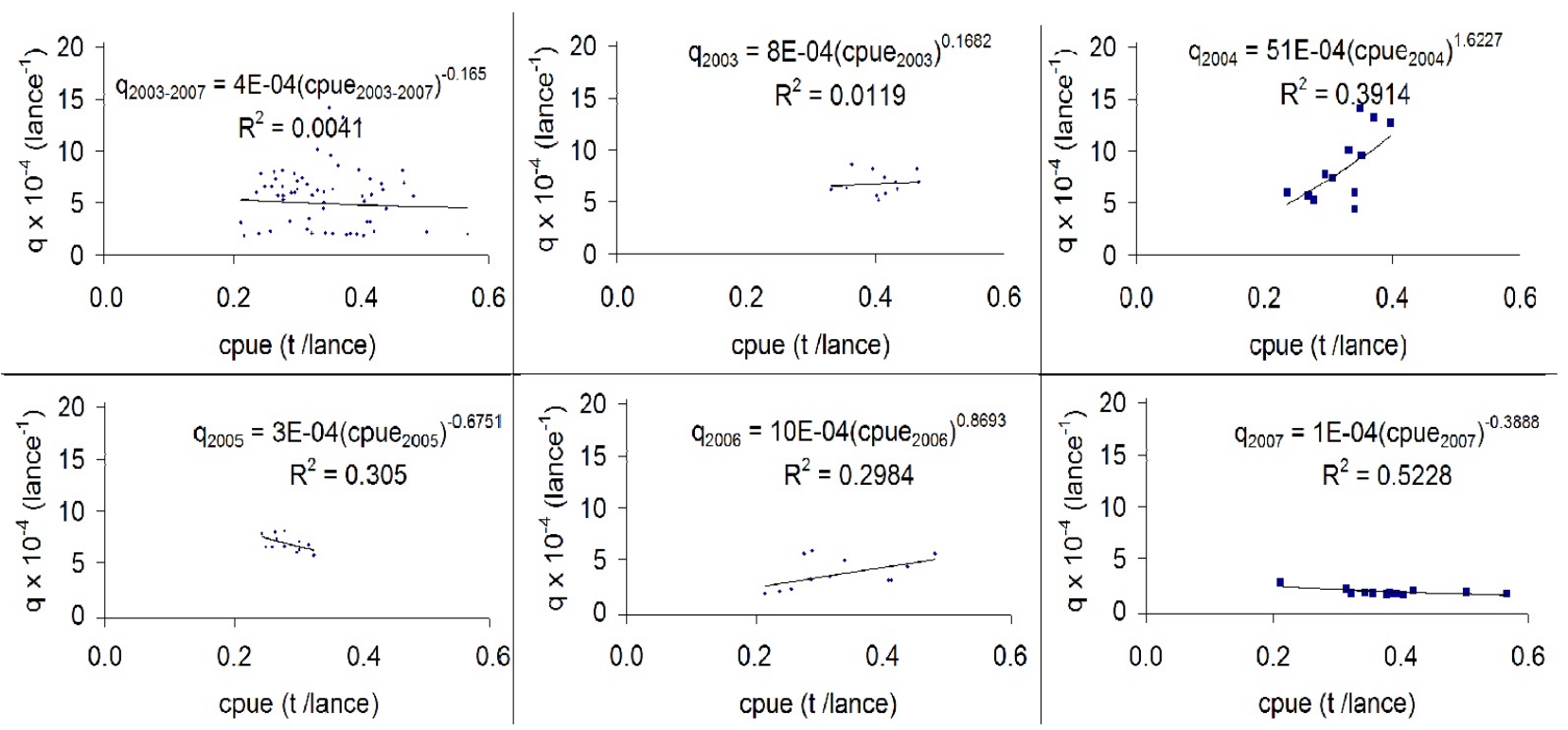

Figura 6. Relación entre la captura por unidad de esfuerzo (cpue) y el coeficiente de capturabilidad (q) mensual en la pesquería de O. remiger en el norte de Perú. Período: enero 2003 a diciembre 2007; n $=60$ meses; $\mathrm{r}^{2}=0,004$. Para cada año, $\mathrm{n}=12$ meses 

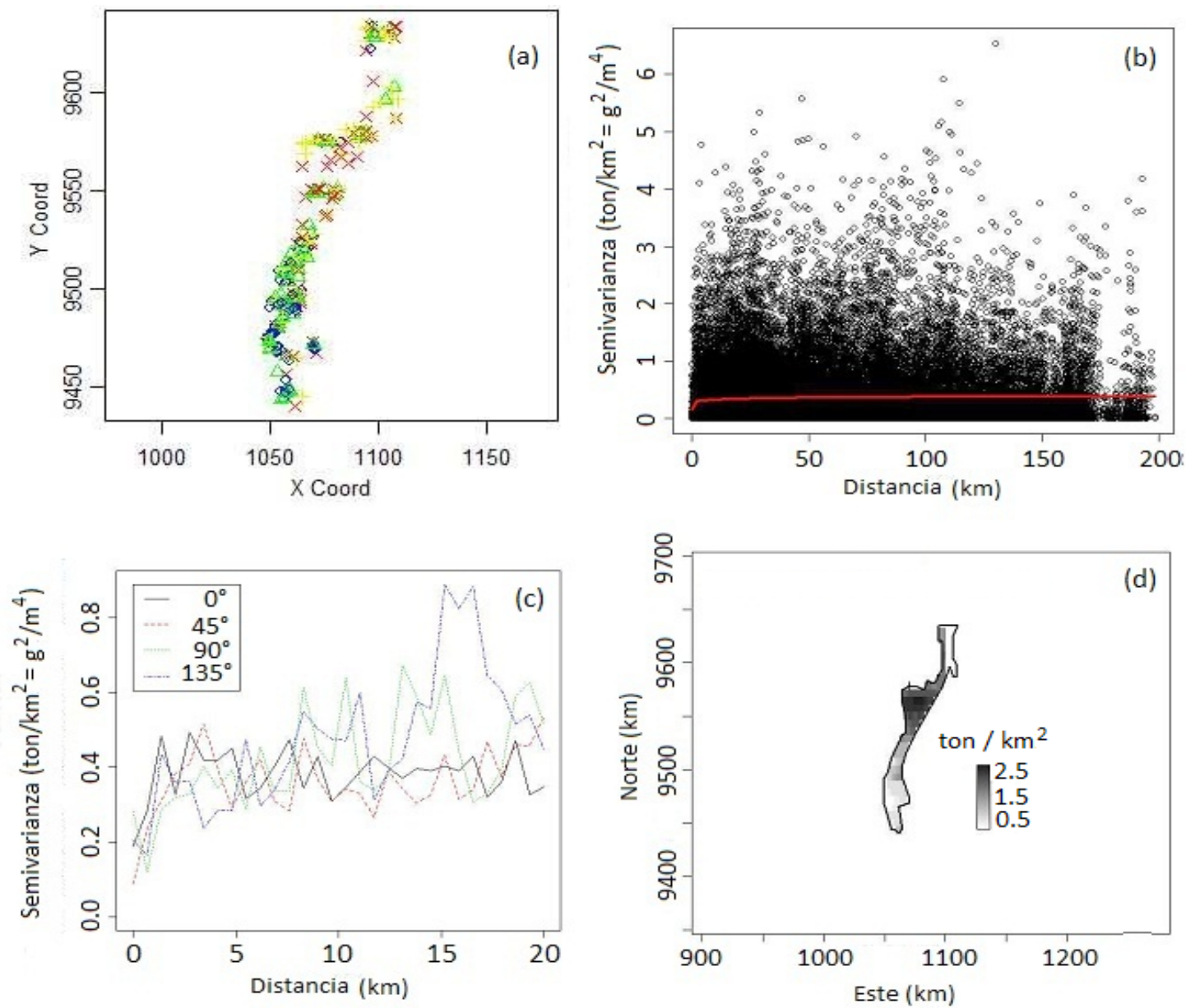

Figura 7. (a) Distribución georeferenciada de lances de pesca, (b) variograma nube, (c) variograma omnidireccional con sensibilidad en cada kilómetro y (d) distribución espacial de densidades en el área de prospección para setiembre del 2007

La tendencia general de la biomasa mensual de $O$. remiger no supera las mil toneladas desde inicios de 2003 hasta el primer trimestre de 2006 (Figura 8 y Tabla 3), cuando la pesquería aun operó al norte de $5^{\circ} \mathrm{S}$.

Tabla 3. Valores máximos y mínimos anuales de biomasa promedio (toneladas) de anguila común por mes en el norte de Perú. Período 2003 a 2007

\begin{tabular}{lrrrrr}
\hline & 2003 & 2004 & 2005 & \multicolumn{1}{c}{2006} & \multicolumn{1}{c}{2007} \\
\hline Máximo & 799,5 & 841,6 & 627,2 & 1448,7 & 2644,5 \\
Mínimo & 423,0 & 273,2 & 345,0 & 506,2 & 791,8 \\
Promedio & 633,5 & 450,1 & 446,0 & 1041,2 & 2034,8 \\
c.v. (\%) & 17,1 & 35,8 & 18,3 & 30,9 & 26,0 \\
\hline
\end{tabular}

La posible tendencia a la baja de la biomasa, que ocurre desde octubre de 2003 hasta setiembre de 2004, podría deberse a que $O$. remiger responde directamente a la pesquería sin alcanzar la capacidad de reemplazo de la biomasa. Luego ocurre un breve incremento de la biomasa mensual (octubre 2004), disminuyendo inmediatamente, permaneciendo cerca de $500 \mathrm{t} / \mathrm{mes}$ o levemente superior (abril 2006). Sin embargo, desde abril 2006 la biomasa mensual de $O$. remiger supera las $1000 \mathrm{t} / \mathrm{mes}$, aunque fluctuante por aumento de la cpue (Figura 5). Notoriamente, desde mayo 2007 la biomasa supera las 2000 t debido a la expansión del caladero al sur de $5^{\circ} \mathrm{S}$.

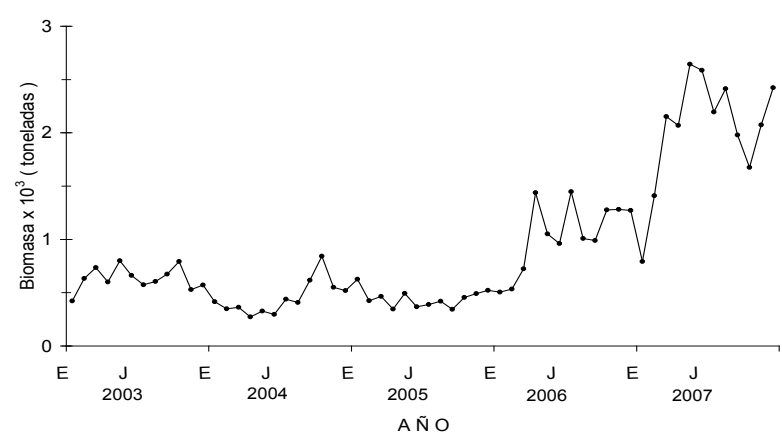

Figura 8. Biomasa mensual de $O$. remiger del norte de Perú en el periodo 2003 a 2007. Período: enero a diciembre $2007 ; n=60$ 


\section{Discusión}

La determinación de variables de una pesquería tales como cpue y biomasa normalmente están sujetas a incertidumbre debido a la calidad de la información base o falta de conocimiento sobre la dinámica de los recursos pesqueros (Punt et al., 1995). Esta imprecisión ha conducido a que la administración pesquera reconozca los peligros de aceptar la estimación de parámetros y su influencia en los resultados obtenidos (Restrepo, 1999). En este sentido, disponer de información de lances geo-referenciados son de mucho valor por su precisión y utilidad en la estimación de variables pesqueras (Arreguín Sánchez, 1996; Walters, 2003; Walters \& Hilborn, 2005), particularmente si la información es obtenida de bitácoras sin sesgos ni errores (e.g. declarar menos capturas, registrar georeferenciadamente el lance de manera inadecuada).

En el período 2003 a 2007, las mayores capturas de $O$. remiger ocurrieron principalmente en el segundo semestre de cada año (excepto en los años 2004 y 2005), lo que difiere con Castillo et al. (2000), quienes señalan que las mayores capturas generalmente ocurren en los meses de invierno y primavera (de julio a septiembre y/o de octubre a diciembre). En los primeros años (2003 a 2005) pudo haber ocurrido el proceso de aprendizaje de las tripulaciones en la pesquería de $O$. remiger, lo que se desprende de observar la trayectoria del coeficiente de capturabilidad, que se incrementó gradualmente desde $6,7 \times 10^{-4}$ lance $^{-1}$, en promedio, en los meses de 2003, alcanzando su máximo valor con 14 x $10^{-4}$ lance $^{-1}$ en abril de 2004 .

La variabilidad en el coeficiente de capturabilidad es muy común en recursos pesqueros (Winters \& Wheeler, 1985; fide Wilberg et al., 2010), como por ejemplo en Heterocarpus reedi en la costa central de Chile (Pérez \& Defeo, 2003); Perca flavescens en el Lago Erie (Jiao et al., 2006) y Gadus morhua en el mar del Norte (Rindorf \& Andersen, 2008), todas ellas especies demersales, como $O$. remiger, o bentónicas. Si la administración pesquera considera siempre a la Ecuación 1 como válida, al incrementarse el coeficiente de capturabilidad mientras la biomasa disminuye, entonces podría conducir a que se sobreestime el tamaño del stock, conduciendo a incrementar el esfuerzo, lo que pudiera causar finalmente, el colapso del recurso (Wilberg et al., 2010). Dado el rol crítico que juega la capturabilidad en la evaluación de los recursos, los modelos deberían considerar la incorporación de su variación espacial y su relación con la cpue para estimar apropiadamente la biomasa de la especie objetivo.

Matsumiya \& Imai(1987) señalan que Conger miriaster, especie demersal del Orden Anguilliformes, presentó la máxima capturabilidad mensual de $30 \times 10^{-4}$ lance $^{-1}$, mientras que en esta investigación $O$. remiger presentó el máximo valor mensual de $14 \times 10^{-4}$ lance $^{-1}$, revelándose, entonces, distintos valores de capturabilidad (en casi el doble) en especies ecológicamente similares (ArreguínSánchez, 1996; Perry et al., 2000). Los mayores valores del coeficiente de capturabilidad de $O$. remiger ocurrieron en abril, mayo y junio de 2004, 2005 y 2006 (promedio: 13×10
${ }^{4}$ lance $^{-1}$; Figura 5), coincidiendo con su mayor actividad reproductiva (Vera, 2006). En al año 2004, la relación entre el coeficiente de capturabilidad y la cpue fue directa y significativa (coeficiente de correlación de Spearman $=$ $0,68 ; p<0,05)$, infiriéndose que en el año 2004 el esfuerzo se orientó a solo una parte del stock, probablemente hacia individuos más vulnerables, coincidiendo con lo reportado para otras especies (Ahrens \& Walters, 2005 y Walters \& Hilborn, 2005), ya que el área efectiva de distribución fue solo de $300 \mathrm{~km}^{2}$, correspondiendo a la menor en todo el periodo analizado (Figura 3). Las diferentes tendencias, ya sean directas o no, en la relación entre cpue y coeficiente de capturabilidad también han sido observados por Marchal et al. (2003) para bacalao del Mar del Norte, Harada et al. (2007) para Eptatretus burgeri y Conger myriaster en la Bahía de Tokio, y Jiao et al. (2006) para Perca flavescens en el lago Erie.

La cpue puede ser proporcional a la densidad local, lo que se conoce como disponibilidad (Walters \& Hilborn, 2005). Esta situación puede conducir a afirmar, equivocadamente que la cpue refleja acertadamente a la biomasa; sin considerar los cambios en la capturabilidad (Casey \& Myers, 1998; Harley et al., 2001; Casini et al., 2005; Wilberg et al., 2010). En el presente estudio, la relación directa entre biomasa y cpue puede encontrarse en el año 2005, en que presentaron tendencias similares ( $p>$ 0,05 ) ya que los valores de capturabilidad se encontraron en un período relativamente estable (Figura 5). La caída de la biomasa de $O$. remiger en los años 2004 y 2005 podría deberse al incremento del esfuerzo, desde 140 hasta 189 lances promedio (Tabla 1), conduciendo a la disminución de la cpue de 0,322 hasta 0,280 t/lance promedio (Tabla 2). Varios estudios de la cpue y biomasa muestran que la distribución espacial de un recurso pesquero en forma de parches es un factor determinante para la evaluación del stock, tal como ha sido informado para Eptatretus burgeri y Conger myriaster de la Bahía de Tokio (Harada et al., 2007), Perca flavescens del Lago Erie (Jiao et al., 2006), y Gadus morhua, Pollachius pollachius, Arnoglossus laterna, Hippoglossoides platessoides y muchas en el Mar del Norte (Casini et al., 2005).

\section{Conclusión}

El presente trabajo revela, gracias al uso de la geoestadística, que en la pesquería de $O$. remiger del norte de Perú la cpue y biomasa mantienen una relación directa en períodos en que la capturabilidad se mantuvo relativamente constante; sin embargo esto no se presenta en la mayor parte del periodo evaluado. Por lo mencionado, se recomienda usar geoestadística para evaluar si la cpue es un buen estimador de la biomasa del recurso, lo que implica considerar su distribución espacial.

\section{Agradecimientos}

Agradezco a mi amigo Mike Rayburn por su desinteresado $\mathrm{y}$ valioso aporte financiero durante la mayor parte del 
tiempo que implicó hacer este estudio. A Rubén Alarcón, por su orientación paciente y didáctica durante el desarrollo de esta investigación. A Rubén Roa-Ureta quien, a pesar de la distancia y sus ocupaciones laborales, brindó su ayuda en varias ocasiones al responder muchos correos electrónicos respecto a las dudas que surgieron durante el proceso geoestadístico. A Roberto Fustos, por compartir sus conocimientos estadísticos para la culminación del presente trabajo. Al profesor Hugo Arancibia por sus revisiones y aportes al presente texto. A Mónica Barros por sus valiosos aportes y consejos en la redacción. A Helen Jutz por su desinteresada paciencia y disponibilidad en la revisión y corrección de los ítems que fueron necesarios traducir.

Al profesor Luís Icochea Salas, por su intervención en la empresa Perupez S.A.C. para contar con toda la valiosa información base de esta publicación. Asimismo, a la empresa Agromar de Pacífico S.A.C. por el financiamiento otorgado en este proyecto.

A Ricardo Bandín y Rodolfo Cornejo, científicos del IMARPE, por las revisiones pertinentes al presente estudio.

\section{Literatura citada}

Ahrens, R \& Walters, C. 2005. Why are there still large pelagic predators in the oceans?. Evidence of severe hyper-depletion in longline catch-per-effort. $1^{\text {st }}$. Meeting of the Scientific Committee of the Western and Central Pacific Fisheries Commission (WCPFCSC1. ME-WP-3). Noumea, New Caledonia. Fisheries Center, University of British Columbia, August 2005, $13 \mathrm{pp}$.

Aitchison, J. 1955. On the distribution of a positive random variable having a discrete probability mass at the origin. J. Am. Stat. Assoc., 50: 901-908.

Alarcón, R. 2003. Evaluación geoestadística del stock de merluza común (Merlucius gayi) utilizando datos de la flota industrial (Tesis para optar el Grado de Magíster en Ciencias, Mención Pesquerías). Universidad de Concepción.

Arreguín-Sánchez, F. 1996. Catchability: A key parameter for fish stock assessment. Reviews in Fish Biology and Fisheries 6: 221-242.

Bahamon, N.; Sardá, F. \& Aguzzi, J. 2009. Fuzzy diel patterns in catchability of deep-water species on the continental margin. ICES Journal of Marine Science 66: 2211-2218.

Cadima, E. 2003. Fish Stock Assessment Manual. Food and Agriculture Organization of the United Nations. Rome, Italy, $181 \mathrm{pp}$.

Casey, J. M. \& Myers, R. A. 1998. Diel variation in trawl catchability: is it as clear as day and night?. Canadian Journal of Fisheries and Aquatic Sciences 55: 23292340.

Casini, M.; Cardinale, M.; Hjelm, J. \& Vitale, F. 2005. Trends in cpue and related changes in spatial distribution of demersal fish species in the Kattegat and Skagerrak, eastern North Sea, between 1981 and 2003. ICES Journal of Marine Science 62: 671-682.

Castillo, R.; Gómez, E. \& Paredes, F. 2000. Pesquería y biología de la anguila común Ophichthus pacifici (Günter, 1870) en el Perú. Informe Progresivo del Instituto del Mar del Perú 134: 3-19.

Castillo, R. \& Gómez, E. 2002. Informe sobre biología, pesquería y utilización de la "anguila común" Ophichthus remiger. Informe del Instituto del Mar del Perú, 16 pp.

Chávez, J. 2000. Análisis dinámico del coeficiente de capturabilidad y sus implicancias en la modelación de pesquerías: Mesodesma donacium en el banco de bahía Coquimbo, un estudio de caso (Tesis. Universidad Católica del Norte), Chile.

Christensen, O. \& Ribeiro, P. 2002. GeoRglm: a package for generalized linear spatial models. R-NEWS 2: 2628.

Dichmont, C.; Punt, A.; Deng, A.; Dell, Q. \& Venables, W. 2003. Application of a weekly delay-difference model to commercial catch and effort data for tiger prawns in Australia's Northern Prawn Fishery. Fisheries Research 65: 335-350.

Diggle, P. J.; Ribeiro, P. J. \& Christensen, O. F. 2003. An introduction to model-based Geo statistics. In Spatial Statistics and Computational Methods, p. 43-86. Ed. by J. Møller. Springer, New York. Lecture Notes in Statistics, 173, $216 \mathrm{pp}$.

Dunn, A.; Harley, S.; Doonan, I. \& Bull, B. 2004. Calculation and interpretation of catch-per-unit-effort (CPUE) indices. Ministry of Fisheries. New Zealand Fisheries Assessment Report, 44 p.

Gaertner, D. \& Dreyfus-León, M. 2004. Analysis of nonlinear relationships between catch per unit effort and abundance in a tuna purse-seine fishery simulated with artificial neural networks. ICES Journal of Marine Science 61: 812-820.

Harada, M.; Tokai, T.; Kimura, M.; Hu, F. \& Shimizu,T. 2007. Size selectivity of escape holes in the coger tube traps for inshore hagfish Eptatretus burgeri and White-spotted coger Conger myriaster in Tokyo Bay. Fisheries Science 73: 477-488.

Harley S. J.; Myers, R. A. \& Dunn, A. 2001. Is catchper-unit-effort proportional to abundance?. Canadian Journal of Fisheries and Aquatic Sciences 58: 17601772.

Horton, N. \& Kleinman, K. 2011. Using R for Data Management, Statistical Analysis, and Graphics. CRC Press. Taylor and Francis Group 79 (2): 285.

IMARPE. 2006. Estado de recursos marinos demersales. Anguila Ophichthus remiger. Recuperado de: http:// www.imarpe.gob.pe

Jiao, Y.; Reid, K. \& Nudds, T. 2006. Variation in the catchability of yellow perch (Perca flavescens) in 
the fisheries of Lake Erie using a Bayesian error-invariable approach. ICES Journal of Marine Science 63: 1695-1704.

Marchal, P.; Ulrich, C.; Korsbrekke, K.; Pastoors, M. \& Rackham, B. 2003. Annual trens in catchability and fish stock assessments. Scientia Marina 67: 63-73.

Matsumiya, Y. \& Imai, M. 1987. Ecology and Abundance of Conger eel Conger myriaster in Shijiki Bay, Hirado Island. Nihon Suisan Gakkaishi. Bulletin of Japanese Society of Science in Fisheries 53 (12): 2127-2131.

Neis, B.; Schneider, D.; Felt, L.; Haedrich, R.; Fischer, J. \& Hutchings, J. 1999. Fisheries assessment: what can be learned from interviewing resource users? Canadian Journal of Fisheries and Aquatic Sciences 56: 1949-1963.

Pérez, E.P. \& Defeo, O. 2003. Time-space variation in the Catchability Coefficient as a function of catch per unit of effort in Hetericarpus reedi (Decapoda, Pandalidae) in north-central Chile. Marine Science 28 (3): 178-182.

Perry, R. I.; Boutillier, J. A. \& Foreman, M. G. 2000. Environmental influences on the availability of smooth pink shrimp, Pandalus jordani, to commercial fishing gear off Vancouver Island, Canada. Fisheries Oceanography 9: 50-61.

PRODUCE, 2008. Resolución Ministerial N486-2008PRODUCE. Publicación electrónica. URL: http:// www.elperuano.pe.

PRODUCE, 2010. Resolución Ministerial N²93-2010PRODUCE. Recuperado de: http://www.elperuano.pe.

PRODUCE, 2016. Evaluación del Plan Operativo III Trimestre 2016. Evaluación del POI - PTI. Recuperado de: http://www.imarpe.gob.pe/imarpe/archivos/ informes/imarpe_evaluac_poi_trim3_2016.pdf $>$

PROMPEX, 2016. Perfil Biológico, Pesquero y Comercial de la Anguila. Gerencia de promoción de mercados del sector pesca y acuicultura.

Punt, A.E.; Butterworth, D.S. \& Martin, J. 1995. The effects of errors in the placement of the boundary between the West and South Coast hake Merluccius spp. stocks on the performance of the current hake management procedure, South African Journal of Marine Science, 15:1, 83-98, DOI: 10.2989/02577619509504835

Quinn, T. \& Deriso, R. 1999. Quantitative Fish Dynamics. Oxford University Press. New York, 542 p.

R Development Core Team. 2008. R: A language and environment for statistical computing. R Foundation for Statistical Computing, Vienna, Austria. ISBN 3-900051-07-0, Recuperado de: http://www.R-project. org.

Regalado, C. \& Ritter, A. 2005. Técnicas geoestadísticas para caracterizar la variabilidad espacial de la hidrofobicidad en la zona no saturada de una cuenca forestal. Instituto Canario Inv. Agrarias (ICIA), Dep. Suelos y Riegos, Apdo. 60 La Laguna, 38200 Tenerife; cregalad@icia.es. Estudios de la Zona No Saturada del
Suelo VII: $235-240$.

Restrepo, V. R., ed. 1999. Proc. 5th Nat'l. NMFS Stock Assessment Workshop: providing scientific advice to implement the precautionary approach under the Magnuson-Stevens Fishery Conservation and Management Act. NOAA Tech. Memo. NMFS-F/SPO40. U.S. Dept. Commerce, Washington, D.C.

Rindorf, A \& Andersen, B. 2008. Do North Sea cod (Gadus morhua) fisheries maintain high catch rates at low stock size? Canadian Journal of Fisheries and Aquatic Sciences 65 (9):1800-1813.

Roa-Ureta, R. \& Niklitschek, E. 2007. Biomass estimation from surveys with likelihood-based geostatistics. ICES Journal of Marine Science 64:1723-1734.

Salthaug, A. \& Aanes, S. 2003. Catchability and the spatial distribution of fishing vessels. Canadian Journal of Fisheries and Aquatic Sciences 60:259-268.

Vera, M. 2006. Talla de primera madurez y época de desove de anguila común Ophichthus remiger en el litoral de la región Tumbes. V Seminario Virtual de las Ciencias del Mar. Centro Regional de Investigación Pesquera y Acuícola - Tumbes. Instituto del Mar del Perú (IMARPE). Recuperado de:http://www.imarpe. gob.pe/tumbes/publicaciones

Walters, C. 2003. Folly and fantasy in the analysis of spatial catch rate data. Canadian Journal of Fisheries and Aquatic Sciences 60: 1433.1436.

Walters, C. \& Hilborn, R. 2005. Exploratory assessment of historical recruitment patterns using relative abundance and catch data. Canadian Journal of Fisheries and Aquatic Sciences 62:1985-1990.

Wilberg, M. J. \& Bence, J. R. 2006. Performance of timevarying catchability estimators in statistical catchat-age analysis. Canadian Journal of Fisheries and Aquatic Sciences 63: 2275-2285.

Wilberg, M.; Thorson, J.; Linton, B. \& Berkson, J. 2010. Incorporating time-varying catchability into population dynamic stock assessment models. Reviews in Fisheries Science 18 (1):7-24.

Winters, G.H. \& Wheeler, J.P. 1985. Interactions between stock area, stock abundance, and catchability coefficient. Canadian Journal of Fisheries and Aquatic Sciences 42: 989-998.

Yousif, A. \& Sabrah, M. 2005. Catchability of the red mullets (Upeneus spp.) from North West red sea during Autum. Egyptian Journal of Acuatic Research 30(B):290-305. 